\title{
NEVANLINNA THEORY AND DIFFERENCE EQUATIONS OF PAINLEVÉ TYPE
}

\section{M.J. Ablowitz and R. Halburd}

\author{
Department of Applied Mathematics, \\ University of Colorado at Boulder, \\ Boulder, CO, 80309-526, USA.
}

Short title: Nevanlinna Theory and Difference Equations

AMS classification scheme: 30D35, 39A10, 39A12.

Keywords: Difference equations, Painlevé, Nevanlinna. 


\begin{abstract}
The complex analytic structure of solutions of difference equations considered to be of Painlevé type is explored. Solutions of a large class of difference equations are meromorphic, modulo singularities due to arbitrary periodic functions. Most of these equations, e.g. the logistic equation, are not integrable and would not be considered to be of Painlevé type. Two tests for difference equations are described. The first checks necessary conditions that non-rational meromorphic solutions are of finite order in the sense of Nevanlinna. The second is a perturbative method similar to Painlevé's $\alpha$-Test in which di-gamma functions play the role of logarithms.
\end{abstract}

\title{
1 Introduction
}

Ordinary differential equations (ODEs) of Painlevé type play an important role in the theory of integrable systems. An ODE is said to be of Painlevé type (or to possess the Painlevé property) if all solutions are single-valued about all movable singularities. In particular, ODEs of Painlevé type arise as symmetry reductions of soliton equations $[4,2,3]$.

The Painlevé property has been used as a powerful detector of integrability since the work of Kowalevskaya in 1889 [11, 12]. Subsequently Painlevé and his colleagues classified all equations of Painlevé type of the form

$$
\frac{d^{2} y}{d z^{2}}=F\left(z ; y, \frac{d y}{d z}\right)
$$

where $F$ is rational in $y$ and $d y / d z$ and (locally) analytic in $z$. All of these equations were solved in terms of known functions except for those that are equivalent (under transformations of the form $z \mapsto \Phi(z)$ and $y \mapsto[a(z) y+$ $b(z)] /[c(z) y+d(z)])$ to one of six ODEs, now called the Painlevé equations $\left(P_{I}-P_{V I}\right)$. The first two Painlevé equations are

$$
\begin{aligned}
P_{I} & \frac{d^{2} y}{d z^{2}}=6 y^{2}+z, \\
P_{I I} & \frac{d^{2} y}{d z^{2}}=2 y^{3}+z y+\alpha,
\end{aligned}
$$

where $\alpha$ is a constant.

Here we describe a number of issues that arise in extending to difference equations the philosophy of using the complex analytic structure of 
solutions as an indication of integrability. We begin by describing the analytic structure of solutions to a class difference equations. We note that, up to arbitrary functions of period one (which play a role akin to that of constants for ODEs and characteristic data for PDEs), general solutions of these difference equations are meromorphic. Hence, for difference equations we encounter the problem of distinguishing well-behaved meromorphic solutions, whereas for differential equations, a meromorphic general solution is sufficient to indicate that the equation is of Painlevé type. We discuss the special class of meromorphic functions that are of finite order of growth in the sense of Nevanlinna.

We also consider a perturbative series expansion method in which the role of the logarithm in standard Painlevé analysis is played by the di-gamma function $\psi(z)=d \log \Gamma(z) / d z$, see also [1].

\section{Complex Difference Equations}

From the dynamical systems standpoint, a discrete equation such as

$$
y_{n+1}=n y_{n}
$$

is viewed as an iterative scheme in which say $y_{1}=k$ is specified and the general solution to equation (3) is $y_{n}=k(n-1)$ !, for all positive integers $n$. In order to extend the Painlevé property to equations such as (3), we wish to understand the analytic structure of solutions in the complex domain, not just at a discrete set of values of the independent variable. To this end we replace equation (3) with the difference (i.e. delay) equation

$$
y(z+1)=z y(z)
$$

which must hold for all $z$ on some Riemann surface. It is straightforward to verify that the general solution of equation (4) is

$$
y(z)=\pi(z) \Gamma(z),
$$

where $\Gamma$ is the gamma function and $\pi$ is an arbitrary function of period one (hereafter we will refer to arbitrary period one functions as "periodic functions"). Periodic functions play an analogous role in the solutions of difference equations to that played by constants in the solutions of differential equations. Since these periodic functions can have any kind of singularity in 
the complex domain, we need to somehow factor them out of our analysis of the complex analytic structure of solutions of difference equations. Apart from possible singularities due to the periodic function $\pi$, the general solution (5) of equation (4) is meromorphic.

On taking the logarithmic derivative of equation (4) it can be seen that the general solution of

$$
\triangle y(z):=y(z+1)-y(z)=\frac{1}{z}
$$

is

$$
y(z)=\psi(z)+\pi(z),
$$

where $\psi(z)=d \log \Gamma(z) / d z$ is the di-gamma function and $\pi$ is an arbitrary periodic function. Equation (6) is important in the theory of difference equations because it is the natural discretization of the equation $d y / d z=1 / z$, which defines the logarithm. The logarithm plays a very special role in Painlevé analysis in that its appearance indicates branching of solutions (logarithms must be introduced in series solutions in which a certain resonance condition is not satisfied). However, note that up to the periodic function $\pi$ the general solution (7) of equation (6) is meromorphic. This indicates that the absence of branching alone does not characterize difference equations of Painlevé type.

In fact, it is known that every equation of the form

$$
y(z+1)=R(y(z))
$$

where $R$ is a rational function of $z$, possesses a non-constant meromorphic solution $y(z)=Y(z)$ (Yanagihara [19]). If $R$ is a polynomial then equation (8) possesses a non-constant entire solution (Shimomura [17], Kimura [10]). Furthermore, a general solution of equation (8) is given by

$$
y(z)=Y(z-\pi(z))
$$

where $\pi$ is an arbitrary periodic function. This implies that the logistic equation

$$
y(z+1)=\mu y(z)(1-y(z))
$$

which has very complicated dynamics for certain values of the parameter $\mu$, has an entire solution. 
In the following we will outline two properties that appear to be necessary for difference equations that one would consider to be of Painlevé type. The first demands that equations of Painlevé type have solutions that satisfy certain growth conditions at infinity. The second approach requires generating series solutions of perturbed equations and demanding that no di-gamma functions appear.

\section{Nevanlinna Theory}

In this section we describe the basic ingredients of Nevanlinna Theory. In particular, we describe the Nevanlinna characteristic function $T(r, f)$, the order of growth of a meromorphic function, and Hadamard's factorization theorem. There are many good references on this subject, such as $[15,8,20$, $13]$.

\subsection{The Nevanlinna Characteristic}

The Nevanlinna characteristic $T(r, f)$, which encodes information about the distribution of values of $f$ on the disk $|z| \leq r$, plays a central role in the theory of meromorphic functions. It is a sum of two parts:

$$
T(r, f)=m(r, f)+N(r, f) .
$$

The proximity function $m(r, f)$ is given by

$$
m(r, f)=m(r, \infty, f)=\frac{1}{2 \pi} \int_{0}^{2 \pi} \log ^{+}\left|f\left(r \mathrm{e}^{i \theta}\right)\right| d \theta,
$$

where $\log ^{+} x=\max \{0, \log x\}$. The proximity function is an averaged measure of how large $f$ becomes on the circle $|z|=r$. Define the counting function $n(r, f)$ to be the number of poles of $f$ (counted according to multiplicities) in the disk $|z|<r$. The integrated counting function, $N(r, f)$, is then defined to be

$$
N(r, f)=N(r, \infty, f)=\int_{0}^{r} \frac{n(t, f)-n(0, f)}{t} d t+n(0, f) \log r .
$$

The Nevanlinna characteristic function given by (9) is therefore the sum of a measure of how large $f$ becomes on $|z|=r$ and a measure of the number of poles of $f$ in $|z|<r$. 
Nevanlinna's First Fundamental Theorem says that for each meromorphic function $f$ and each complex number $a$,

$$
\left|T\left(r, \frac{1}{f-a}\right)-T(r, f)\right|
$$

is bounded as $r$ approaches infinity. The Nevanlinna characteristic $T\left(r, \frac{1}{f-a}\right)$ measures the affinity of $f$ for the value $a$ in that it is the sum of the proximity function $m(r, a, f):=m\left(r, \frac{1}{f-a}\right)$, which measures how close $f$ stays to the value $a$ on the circle $|z|=r$, and the integrated counting function $N(r, a, f):=N\left(r, \frac{1}{f-a}\right)$, which is a measure of the number of times $f=a$ in the disk $|z|<r$.

Nevanlinna's First Fundamental Theorem says that the affinity of $f$ for any value is essentially invariant in that the difference between the affinities for any two values is bounded. Let us consider the content of this theorem for the function $f(z)=\mathrm{e}^{z}$. A straightforward calculation gives $T\left(r, \mathrm{e}^{z}\right)=r / \pi$. Since $\mathrm{e}^{z}$ is never zero or infinity, $N\left(r, 0, \mathrm{e}^{z}\right)=N\left(r, \infty, \mathrm{e}^{z}\right)=0$. For all other values of $a, N\left(r, a, \mathrm{e}^{z}\right) \rightarrow \infty$ as $r \rightarrow \infty$, so $m\left(r, 0, \mathrm{e}^{z}\right)$ and $m\left(r, \infty, \mathrm{e}^{z}\right)$ must be larger than $m\left(r, a, \mathrm{e}^{z}\right)$ for $a \neq 0, \infty$.

Recall that $m(r, a, f)$ is a measure of how close $f(z)=\mathrm{e}^{z}$ stays to the value $a$ on the circle $|z|=r$. So Nevanlinna's First Fundamental Theorem says that since $\mathrm{e}^{z}$ does not take the values 0 and $\infty$, it must take values near 0 or $\infty$ on large parts of the circle $|z|=r$ for large $r$. This is reflected in the fact that $\mathrm{e}^{z} \rightarrow 0$ as $\Re\left(\mathrm{e}^{z}\right) \rightarrow-\infty$ and $\mathrm{e}^{z} \rightarrow \infty$ as $\Re\left(\mathrm{e}^{z}\right) \rightarrow+\infty$.

\subsection{The Order of a Meromorphic Function}

The Nevanlinna characteristic leads to a natural measure of the rate of growth of a meromorphic function. Specifically, the order of growth of a meromorphic function $f$ is defined to be

$$
\sigma(f)=\limsup _{r \rightarrow \infty} \frac{\log T(r, f)}{\log r} .
$$

If $f$ is entire then this reduces to the usual definition of growth, namely,

$$
\sigma(f)=\limsup _{r \rightarrow \infty} \frac{\log \log M(r, f)}{\log r},
$$


where

$$
M(r, f)=\max _{|z|=r}|f(z)| .
$$

In particular

$$
\sigma\left(\exp \left(a_{0}+a_{1} z+\cdots+a_{n} z^{n}\right)\right)=n, \quad\left(a_{n} \neq 0\right) .
$$

Most meromorphic functions have infinite order. The class of finite-order meromorphic functions possess some remarkable properties. In particular, such functions possess the following factorization:

\section{Hadamard's Factorization Theorem}

Let $f$ be a meromorphic function such that

$$
\lim _{r \rightarrow \infty} r^{-k-1} T(r, f)=0,
$$

where $k$ is a non-negative integer. Let $a_{1}, a_{2}, \ldots$, and $b_{1}, b_{2}, \ldots$, be the zeros and poles of $f$ respectively (other than at $z=0$ ) repeated according to multiplicity. Let $h$ be the multiplicity of any zero (or pole for $h<0$ ) at $z=0$. Then there is a polynomial of degree no greater than $k$ such that

$$
f(z)=z^{h} \mathrm{e}^{P(z)} \lim _{R \rightarrow \infty} \frac{\prod_{\left|a_{i}\right|<R} E\left(\frac{z}{a_{i}}, k\right)}{\prod_{\left|b_{i}\right|<R} E\left(\frac{z}{b_{i}}, k\right)},
$$

where

$$
E(\zeta, k)= \begin{cases}(1-\zeta) \exp \left(\zeta+\frac{\zeta^{2}}{2}+\cdots \frac{\zeta^{k}}{k}\right), & k \neq 0 \\ 1-\zeta, & k=0\end{cases}
$$

is the Weierstrass primary factor of order $k$.

Hadamard's Factorization Theorem shows that any finite-order meromorphic function is defined up to a polynomial by its poles and zeros.

\subsection{Finite-Order Solutions of Differential and Differ- ence Equations}

Let

$$
R_{p, q}(z ; y)=\frac{a_{0}(z)+a_{1}(z) y+\cdots+a_{p}(z) y^{p}}{b_{0}(z)+b_{1}(z) y+\cdots+b_{q}(z) y^{q}}
$$


where the $a_{j}$ and $b_{j}$ are meromorphic and $a_{p}, b_{q}$ are both non-zero. Any differential equation of the form

$$
\frac{d y}{d z}=R_{p, q}(z ; y(z))
$$

with a meromorphic general solution must possess the Painlevé property and therefore must be a Riccati equation, i.e. $p \leq 2$ and $q=0$. In fact, Malmquist [14] showed that any equation of the form (11), where the $a_{j}$ 's and $b_{j}$ 's are rational, that admits a non-rational meromorphic solution must be a Riccati equation. Subsequently Wittich [18] showed that all meromorphic solutions of Riccati equations with rational coefficients $a_{j}$ are of finite order. Furthermore, the Jacobi and Weierstrass elliptic functions, which satisfy firstorder equations of the form

$$
\left(\frac{d y}{d z}\right)^{2}=P(y)
$$

where $P$ is a polynomial of degree three or four, are of order two [8].

Moving on to second-order differential equations, we note that of the Painlevé equations $P_{I}-P_{V I}$, only $P_{I}, P_{I I}$, and $P_{I V}$ have meromorphic general solutions. The order of the non-rational solutions of these equations are $5 / 2$, 3 , and 4 respectively $[5,8]$. These results suggest that meromorphic solutions to equations of Painlevé type are of finite order.

We consider conditions on the form of certain rational difference equations that are necessary if the equation is to admit a meromorphic solution of finite order. Yanagihara [19] considered the first-order difference equation

$$
y(z+1)=R_{p, q}(z ; y(z)),
$$

where $R_{p, q}$ is given by (10) in which the $a_{j}$ 's and $b_{j}$ 's are taken to be rational. He showed that if this equation admits a non-rational meromorphic solution of finite-order then $\max (p, q)=1$. That is, the equation is the (linearizable) difference Riccati equation. In Ablowitz, Halburd, and Herbst [1] the techniques of Yanagihara were used to show that the only second-order difference equations of the form

$$
y(z+1)+y(z-1)=R_{p, q}(z ; y(z)) \quad \text { or } \quad y(z+1) y(z-1)=R_{p, q}(z ; y(z)),
$$

that admit finite-order non-rational meromorphic solutions, satisfy $\max (p, q) \leq$ 2. We remark that this class of difference equations includes

$$
y(z+1)+y(z-1)=\frac{(\alpha z+\beta) y(z)+\gamma}{1-y^{2}(z)},
$$


where $\alpha, \beta$, and $\gamma$ are constants. Equation (12) is a known discretization of the second Painlevé equation which is considered to be integrable (see, e.g., [16]).

From the above it follows that any meromorphic solution of the difference equation

$$
y(z+1)+y(z-1)=y(z)+\frac{a}{y^{2}(z)},
$$

where $a$ is a non-zero constant, necessarily has infinite order and so we would not consider it to be of Painlevé type. Hietarinta and Viallet [7] pointed out that despite the fact that equation (13) possesses the singularity confinement property, a property of discrete (i.e. lattice) equations considered to be related to the Painlevé property [6], numerical calculations suggest that it is chaotic.

\section{Series Methods}

The second method that we describe here is related to Painlevé's $\alpha$-test (see Ince [9] for a description of Painlevé's $\alpha$-Test). It involves adding a perturbation, whose size is determined by a small parameter $\epsilon$, to a known integrable difference equation and expanding the solution as a series in $\epsilon$. In this test, the di-gamma function $\psi(z)=d \log \Gamma(z) / d z$ plays a role analogous to that of the logarithm in Painlevé analysis. That is, the expansions generated from an equation of Painlevé type should not contain di-gamma functions.

In [1] the equation

$$
y(z+1)+y(z-1)=\frac{(2+\epsilon f(z)) y(z)}{1-y^{2}(z)}
$$

was considered, where $f$ is an analytic function. Substituting the expansion

$$
y(z)=y_{0}(z)+\epsilon y_{1}(z)+O\left(\epsilon^{2}\right)
$$

into equation (14) and equating the coefficients of different powers of $\epsilon$ to zero constrains $y_{0}$ and $y_{1}$. In particular, $y_{0}$ satisfies

$$
y_{0}(z+1)+y_{0}(z-1)=\frac{2 y_{0}(z)}{1-y_{0}^{2}(z)},
$$


whose general solution is given in terms of elliptic functions, and $y_{1}$ satisfies the linear difference equation

$$
y_{1}(z+1)-2 \frac{\left(1+y_{0}^{2}(z)\right)}{\left(1-y_{0}^{2}(z)\right)^{2}} y_{1}(z)+y_{1}(z-1)=\frac{y_{0}(z) f(z)}{1-y_{0}^{2}(z)} .
$$

It can be shown [1] that $y_{1}$ necessarily contains a factor proportional to a di-gamma function (i.e. $\psi(z-\pi(z)))$ unless $f$ satisfies

$$
f(z+1)-2 f(z)+f(z-1)=0 .
$$

That is, unless $f(z)=\alpha z+\beta$, where $\alpha$ and $\beta$ are periodic functions. Taking $\alpha$ and $\beta$ to be constants, we recover the standard (integrable?) difference version of $P_{I I}$, equation (12) with $\gamma=0$.

\section{Discussion}

Two approaches to the extension of the Painlevé property to difference equations have been considered. In the first, we demand that non-rational meromorphic solutions be of finite order in the sense of Nevanlinna. In the second, we consider series expansions of solutions and demand that they be free of di-gamma functions.

\section{References}

[1] M.J. Ablowitz, R Halburd, and B Herbst. On the extension of the Painlevé property to difference equations. U. Colorado APPM Report no. 403, 1999.

[2] M.J. Ablowitz, A. Ramani, and H. Segur. Nonlinear evolution equations and ordinary differential equations of Painlevé type. Lett. Nouvo Cim., 23:333-338, 1978.

[3] M.J. Ablowitz, A. Ramani, and H. Segur. A connection between nonlinear evolution equations and ordinary differential equations of P-type. I and II. J. Math. Phys., 21:715-721, 1006-1015, 1980.

[4] M.J. Ablowitz and H. Segur. Exact linearization of a Painlevé transcendent. Phys. Rev. Lett., 38:1103-1106, 1977. 
[5] P. Boutroux. Sur quelques propriétés des fonctions entières. Acta Math., 28:97-224, 1904.

[6] B. Grammaticos, A. Ramani, and V. Papageorgiou. Do integrable mappings have the Painlevé property? Phys. Rev. Lett., 67:1825-1828, 1991.

[7] J. Hietarinta and C. Viallet. Singularity confinement and chaos in discrete systems. Phys. Rev. Lett., 81:325-328, 1998.

[8] E. Hille. Analytic Function Theory. Ginn, Boston, 1959-62. vols 1-2.

[9] E.L. Ince. Ordinary Differential Equations. Dover, New York, 1956.

[10] T. Kimura. On the iteration of analytic functions. Funkcial. Ekvac., 14:197-238, 1971.

[11] S. Kowalevski. Sur le problème de la rotation d'un corps solid autour d'un point fixè. Acta Math., 12:177-232, 1889.

[12] S. Kowalevski. Sur une propriété d'un système d'équations différentielles qui definit la rotation d'un corps solide autour d'un point fixè. Acta Math., 14:81-93, 1889.

[13] I. Laine. Nevanlinna theory and complex differential equations. Walter de Gruyter \& Co., Berlin, 1993.

[14] J. Malmquist. Sur les fonctions á un nombre fini des branches définies par les équations différentielles du premier ordre. Acta Math., 36:297343, 1913.

[15] R. Nevanlinna. Analytic functions. Springer-Verlag, New York, 1970. Translated from the second German edition by Phillip Emig. Die Grundlehren der mathematischen Wissenschaften, Band 162.

[16] A. Ramani, B. Grammaticos, and J. Hietarinta. Discrete versions of the Painlevé equations. Phys. Rev. Lett., 67:1829-1832, 1991.

[17] S. Shimomura. Entire solutions of a polynomial difference equation. $J$. Fac. Sci. Univ. Tokyo Sect. IA Math., 28:253-266, 1981.

[18] H. Wittich. Einige Eigenschaften der Lösungen von $w^{\prime}=a(z)+b(z) w+$ $c(z) w^{2}$. Arch. Math., 5:226-232, 1954. 
[19] N. Yanagihara. Meromorphic solutions of some difference equations. Funkcial. Ekvac., 23:309-326, 1980.

[20] Guan-Hou Zhang. Theory of Entire and Meromorphic Functions: Deficient and Asymptotic Values and Singular Directions. AMS, Providence, Rhode Island, 1993. Translations of Mathematical Monographs, v. 122. 